\title{
Tuberculous brain stem abscesses in children
}

\author{
Raj Kumar ${ }^{1}$, Vinita Singhi ${ }^{2}$ \\ Departments of ${ }^{1}$ Neurosurgery, ${ }^{2}$ Neuroanaesthesiology Sanjay Gandhi Postgraduate \\ Institute of Medical Sciences and C.S.M. Medical University, Lucknow, India
}

\begin{abstract}
Tuberculous brain stem abscess is a highly uncommon entity. Three children with tuberculous brain stem abscesses of 1 month, 7 and 12 years of age were treated by microsurgical evacuation and biopsy of capsule, utilizing safe incisions over dorsal brain stem, through the floor of $I V^{\text {th }}$ ventricle. There was no added deficit postoperatively in these children. Microsurgical evacuation and biopsy helped in differentiating these lesions from pyogenic abscesses in two cases for appropriate treatment. The third operated case was a child, who while being on antituberculosis treatment for proven tuberculous meningitis for $\mathbf{1 . 5}$ year developed medullary and pontine abscess and tuberculoma exemplifying a paradoxical response to chemotherapy. Brain stem abscess especially tuberculous need surgical drainage, as it provides an accurate proof of offending organism and also takes care of a paradoxical response to antituberculosis chemotherapy in diagnosed cases. The safe incisions on brain stem are helpful to avoid the morbidity. (J Pediatr Neurol 2004; 2(2): 101-106).
\end{abstract}

Key words: brain stem abscess, tuberculosis.

\section{Introduction}

The brain stem is an uncommon site for abscess to occur. It accounts for only $0.5-6 \%$ of brain abscesses (1) while, tuberculous abscess is further

Correspondence: Dr. Raj Kumar

Associate Professor

Department of Neurosurgery

Sanjay Gandhi Post Graduate Institute of

Medical Sciences Lucknow - 226014, U.P. INDIA.

Tel: 0 522-2668700, fax: 91 (522) 2668129.

E-mail: rajkumar@sgpgi.ac.in

Received: December 30, 2003.

Revised: January 20, 2004.

Accepted: January 21, 2004. rare in brain stem. It is an infrequently recognized entity. This together with an old neurosurgical dogma, that brain stem lesions were inoperable, contributed to the uniformly fatal outcome of this disease. The better understanding of microsurgical anatomy and different microsurgical approaches to each segment of brain stem has resulted in few case reports of successful outcome. The best and safe method of treatment for brain stem abscess is yet to be defined. It can be managed by medical treatment alone, stereotactic aspiration of pus and medical treatment, or surgical excision/drainage of the abscess. Here we are presenting three children of brain stem tuberculous abscesses, who were treated surgically along with antituberculosis chemotherapy. The clinical profile, microsurgical incisions over brain stem for drainage, and outcome of these children is discussed here.

\section{Case Reports}

Case 1

1-month-old female child presented to us with intermittent fever for 15 days, deviation of angle of mouth to left for 15 days and weakness of left side of body for 4 days. On examination she was conscious, alert, following light and sound, had right $\mathrm{VI}^{\text {th }}$ and VII ${ }^{\text {th }}$ nerve paresis. She had left sided hemiparesis (muscle power 3/5) with extensor plantar response. On investigation, her hemoglobin was $(9.2 \mathrm{~g} / \mathrm{dL})$, total leukocyte count $7600 / \mathrm{mm}^{3}$. Contrasted computed tomography (CT) of head showed a ring enhancing lesion with thick ring, placed eccentrically to right in pons with its extension into upper medulla $(2.5 \times 2 \mathrm{~cm}$ in size) with perilesional edema. It had a daughter nodular ring projecting posteriorly in 4th ventricle (Figure 1). A clinico-radiological diagnosis of pyogenic pontine abscess was considered. Midline suboccipital craniectomy and drainage of abscess was done through the floor of $\mathrm{IV}^{\text {th }}$ ventricle by suprafacial incision on right side. Yellowish thick pus (approximately $6 \mathrm{ml}$ ) was drained out and a part of abscess capsule was taken for biopsy. Histopathological report revealed granulomatous inflammatory pathology suggestive of tuberculosis. The culture of pus did not reveal either pyogenic 


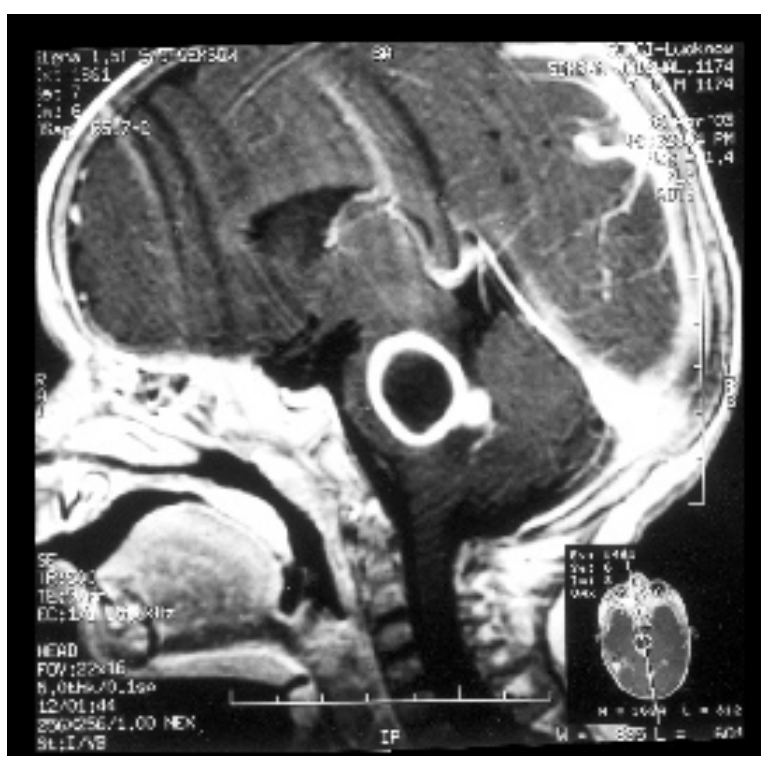

Figure 1. Sagittal section of contrast T1wt MRI showing ring enhancing lesion with thick ring, mainly in pons, an adjacent nodule is seen dorsally.

or acid fast organism. The child was further investigated to search the primary site of infection following the histological diagnosis of tuberculosis, but the tests remained inconclusive. Post-operative period was uneventful; fever subsided on fourth post-operative day. Repeat CT scan showed complete resolution of abscess (Figure 2). Right sided $\mathrm{VI}^{\text {th }}$ and $\mathrm{VII}^{\text {th }}$ nerve, and left sided hemiparesis improved significantly. The child was kept on four drugs antituberculosis regimen (rifampicin, streptomycin, isoniazide, and pyrazinimide) with a plan to continue it for 8 months followed by two drugs to complete the $1 \frac{1}{2}$ year schedule. At the follow up of 4 months the child had no deficit other than restriction of right sided lateral gaze.

\section{Case 2}

Seven years old female child presented to us with unsteady gait and swaying to either side on walking for 1 month, hoarseness of voice and difficulty in deglutition for 20 days, weakness of left side of body with altered sensorium for 15 days and fever for 4 days. On clinical examination she was unconscious with occasional eye opening to pain, not verbalizing or following commands and having flexor response to pain. Pupils were normal in size and reaction and $\mathrm{VII}^{\text {th }}$ nerve paresis of lower motor neuron type was evident on both sides. On lower cranial nerve examination, bilateral gag reflex was impaired. She had generalized spasticity with grade II power on left side and grade III on the right sided limbs. Deep tendon reflexes were normal on both sides and plantar were extensor. There were no meningeal signs. Examination of chest revealed

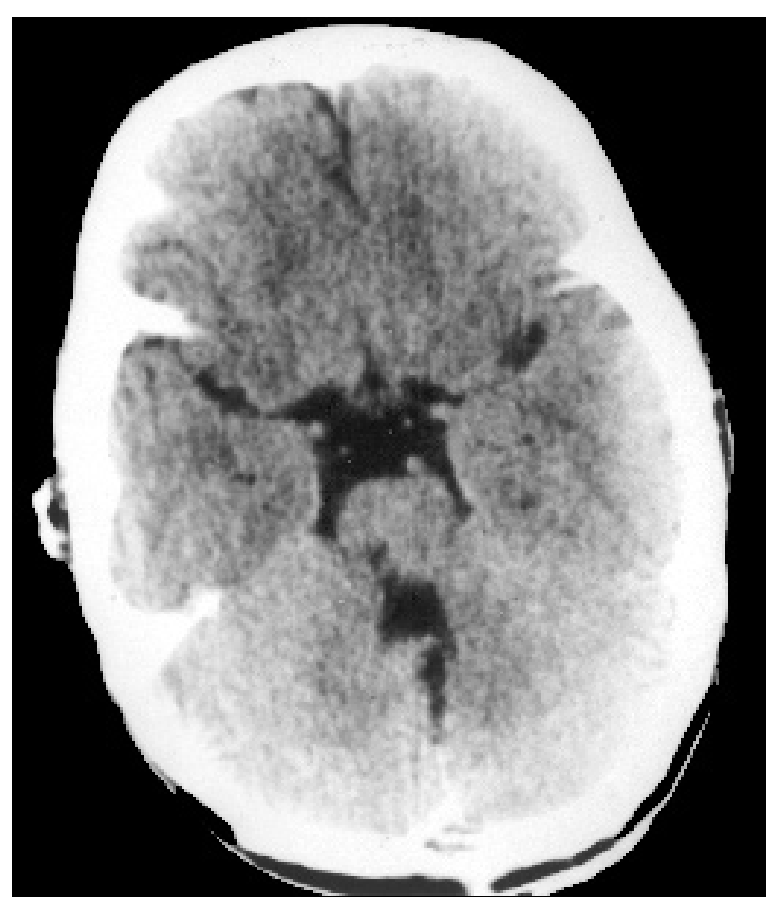

Figure 2. Post-operative CT head of same child (Figure 1) showing postoperative changes at the site of surgery, No abscess is seen.

bilateral basal crepitations. Routine investigations including erythrocyte sedimentation rate were normal. Contrasted CT of head showed two well defined ring enhancing mass lesions in midbrain and pons with central hypodensity. These were pointing dorsally to obliterate the $\mathrm{IV}^{\text {th }}$ ventricle. Considering the diagnosis of brain stem abscess a midline suboccipital craniectomy and trans IV $^{\text {th }}$ ventricular evacuation of abscess was done through midline above facial colliculus incision. Brain stem was bulging posteriorly, and on making an opening at dorsally pointing wall of abscess in floor of $I^{\text {th }}$ ventricle a purulent fluid came out which was collected for culture and sensitivity. Postoperatively she developed cerebrospinal fluid (CSF) leak from the stitch line which was treated by lumbar drainage twice a day for 5 days. CSF examinations revealed cells of $12 / \mathrm{mm}^{3}$, all lymphocytes, protein $1510 \mathrm{mg} / \mathrm{L}$, glucose $41 \mathrm{mg} / \mathrm{dL}$ and a sterile culture. CSF examination was pointing towards tuberculosis, though staining and cultures of pus were unremarkable. Histopathological examination of capsule showed granulomatous inflammatory pathology. The patient was placed on broad spectrum antibiotics. At the time of discharge on $10^{\text {th }}$ day, the condition of the patient was same as before surgery. In view of lack of clinical improvement, he was started on emperical antituberculosis chemotherapy with four drugs regimen to which she responded dramatically and improved significantly within 2 weeks following antituberculosis chemotherapy. At follow-up to 3.5 months, she was conscious, alert 

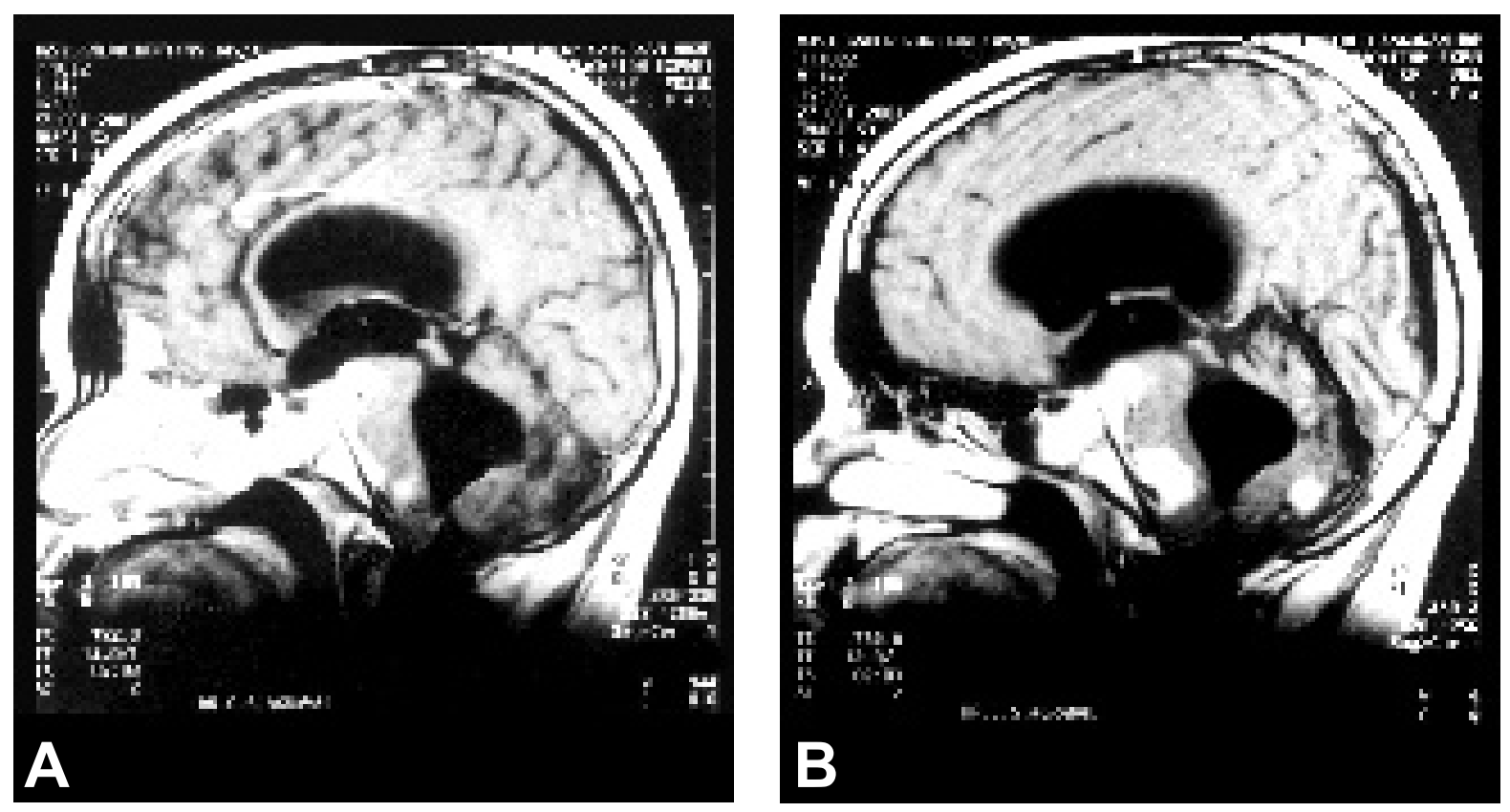

Figures 3a and 3b. Sagittal contrast T1wt MR images depicting hyperintense granuloma in ponse and medulla. A small area of hypointensity can be seen in medulla (an area of abscess). A good ring is not seen in these images. Ventricular dilation is seen.

and talking, and had residual hemiparesis of grade III. Bilateral subtle VII ${ }^{\text {th }}$ nerve paresis was persisting. Screening for systemic tuberculosis in this child was also negative at first follow-up of 4 weeks.

\section{Case 3}

Twelve years male, a diagnosed and operated case of post tuberculous meningitis, hydrocephalus, who had undergone shunt revisions twice during last one year presented to us with signs of raised intracranial pressure, nasal regurgitation of fluids and hoarseness of voice for 3 weeks. He became dysphagic for liquids and then solids during last 2 weeks. Parents gave history of generalized swelling of body, loss of appetite, lethargy, and paraparesis for 1 weeks and facial asymmetry for 5 days. Clinical examination revealed normal higher mental functions but sluggish response. Vision on right eye was hand movements at 2 feet and no perception of light from left eye, the right pupil was reacting to light, but there was no reaction on left side. Extraocular movements were full. Fundoscopic examination showed secondary optic atrophy on both sides. There was left sided VII ${ }^{\text {th }}$ nerve paresis of lower motor neuron type. There was profound sensorineural hearing loss on left side (VIII ${ }^{\text {th }}$ nerve paresis). Bilateral gag reflex was impaired (IX ${ }^{\text {th }}$ and $\mathrm{X}^{\text {th }}$ nerve paresis), XI nerve was bilaterally normal and XII ${ }^{\text {th }}$ nerve paresis was present on left side. No cerebellar signs were present on gross testing. In both the lower limbs tone was increased with muscle power $4 / 5$, while in upper limbs muscle power was $5 / 5$. Deep tendon reflexes were brisk bilaterally, ankle clonus was present on both side and bilateral plantar responses were extensor. On investigation hemoglobin was $10.5 \mathrm{~g} / \mathrm{dL}$, leukocyte count $8300 / \mathrm{mm}^{3}$, erythrocyte sedimentation rate $(45 \mathrm{~mm} /$ hour$)$. Contrasted CT scan of head showed right ventriculoperitoneal shunt in situ with dilated ventricular system and patchy areas of heterodense enhancement on the left side of mid brain, pons and left medial temporal lobe with minimal enhancement of basal cisterns. Magnetic resonance imaging of head revealed hypointense on T1wt and hyperintense on T2wt lesions occupying the left side of the brain stem at the level of lower midbrain pons and medulla and left side of the temporal lobe. The entire lesion was enhancing on contrast and there was a small ring enhancement with central hypointensity in medulla (Figures $3 \mathrm{a}$ and $3 \mathrm{~b}$ ). Considering the diagnosis of tuberculoma in pons and liquefying tuberculoma in medulla, a sub-occipital craniectomy and decompression of brain stem lesion was attempted through left suprafacial approach. On operation lesion appeared whitish yellow, solid firm, non vascular and non suckable mass. First a shunt was revised and later a sub total excision of lesion was done. In medulla an abnormal tissue was visualized which on opening through midline incision yielded 2 cc of frank pus, which turned out to be positive for acid fast staining. Post operative period was uneventful. No fresh deficit appeared following surgery and general condition also improved. 
Biopsy of the tissue revealed granulomatous inflammation suggestive of tuberculosis etiology. He was already receiving three drugs regimen of antituberculosis therapy (rifampicin, isoniazid, and pyrazinamide) for 1.5 years following the diagnosis of tuberculous meningitis. He was put on second line antituberculosis drugs on appropriate doses (ciprofloxacin and cycloserine) along with rifampicin and isoniazid. At 1.5 months followup his father informed that his son was gradually improving but still bed ridden on Ryle's tube feeds.

\section{Discussion}

Brain abscesses account for $1 / 10,000$ hospitalizations in general (2), an incidence of 0.13 per 100,000 people has been calculated (3). Brain stem sites account for only $0.5-6 \%$ of all brain abscesses (1). Clinical signs of a brain stem abscess develop quickly, enabling early diagnosis while the lesion is small. The mean age of patients in literature is 37 years, but our children were 1 month, 7 years to 12 years aged, signifying no age is exempted for brain stem abscess to occur. Very few case reports on brain stem abscesses are available $(4,5)$. Tuberculous abscesses are even rarer entity. Moreover there has been no case report on brain stem abscess in infant as operated by us (Case 1). These abscesses are most often pontine and mesencephalic, rarely medullary (6). Though one of our cases had small abscess in medulla with granuloma in rest of brain stem on one side. The child had dense deficit on account of medullary lesions. Most pyogenic brain stem abscesses develop either by hematogenous metastasis from remote foci of infection, usually in the lungs, or by direct extension from an adjacent structure. It is remarkable that the infective source remains unknown in $37 \%$ of brain stem abscess, compared with $10-18 \%$ of brain abscess in other locations (7). Most common organisms in brain stem abscess are streptococcus and staphylococcus species with other gram negative and anaerobic bacteria accounting for most other cases. Mycobacterium tuberculosis is highly uncommon cause of brain stem abscess and usually results from liquefaction of a tuberculoma (8). It should be considered in a region where pulmonary disease is widely prevalent like in India because central nervous system tuberculosis is always secondary to some primary infection elsewhere in body. The spread occurs by hematogenous route, though our two cases did not show any stigmata suggestive of either pulmonary or other primary tuberculosis at their first presentation. Such presentations suggest that the possibility of tuberculosis etiology in an abscess can not be ruled out, even if there is no evidence of primary tuberculosis in children. Our third case was a known case of tuberculous meningitis and hydrocephalus, which had been treated with regular antituberculosis drugs along with ventriculoperitoneal shunt. In spite of this he developed new lesions (tuberculoma in the left temporal lobe, brain stem abscess), which could be due to the resistant or atypical mycobacteria prevalent in this subcontinent. However, the paradoxical increase in size of pre-existing tuberculomas or appearance of new granulomas, despite regular antituberculosis chemotherapy is well established phenomenon that had been reported by principal author previously (9). However, it was surprising to have an additional abscess other than granuloma in the third child despite a regular course of chemotherapy.

Radiologic and other diagnostic tests are unable to distinguish between tuberculous and bacterial brain abscesses (10). Tuberculous abscess may be unilocular or multilocular. A relatively long clinical history, together with an appearance of enhanced capsule with a thick wall on CT may clinch the diagnosis (11). However, by definition a tuberculous abscess is an encapsulated collection of pus containing viable tuberculous bacilli without evidence of classical tubercular granuloma (11, 22). According to old diagnostic criteria's of tubercular abscess by Whitner (8), (i) Microscopic evidence from surgical or autopsy material of true abscess formation and within the brain substance characterized by cavity formation and central pus. (ii) Sufficient histological description to ensure that the inflammatory reaction in the abscess wall is predominantly a vascular granulation tissue containing acute and chronic inflammatory cells, particularly polymorphonuclear leukocytes. (iii) Proof of tuberculous origin by either a positive pus culture for Mycobacterium tuberculosis or by demonstration of acid-fast bacilli in the pus or abscess wall.

Above definition and criteria's are controversial today, because it is difficult to recover the viable tubercular bacilli from pus, or CSF, or sometimes from other body fluids of other system's tuberculosis. It seems to be illogical to satisfy these criteria's in all cases especially when the sample size is small. Secondly, if the described wall of abscess is thick in tuberculous abscess, then a granulomatous reaction is always a possibility. Mycobacteria are only infrequently isolated from the CSF and pus perhaps due to the small number of organisms in the CSF. Polymerase chain reaction is a rapid, sensitive, and specific diagnostic method in CSF of immunocompromised patients with tuberculous meningitis (12), though the value of this technique in the diagnosis of tuberculous abscess needs evaluation. Lumbar puncture is hazardous in patients with brain abscess (13), and the pathogen often can not be determined from CSF (14). The differentiation between tuberculous and pyogenic 


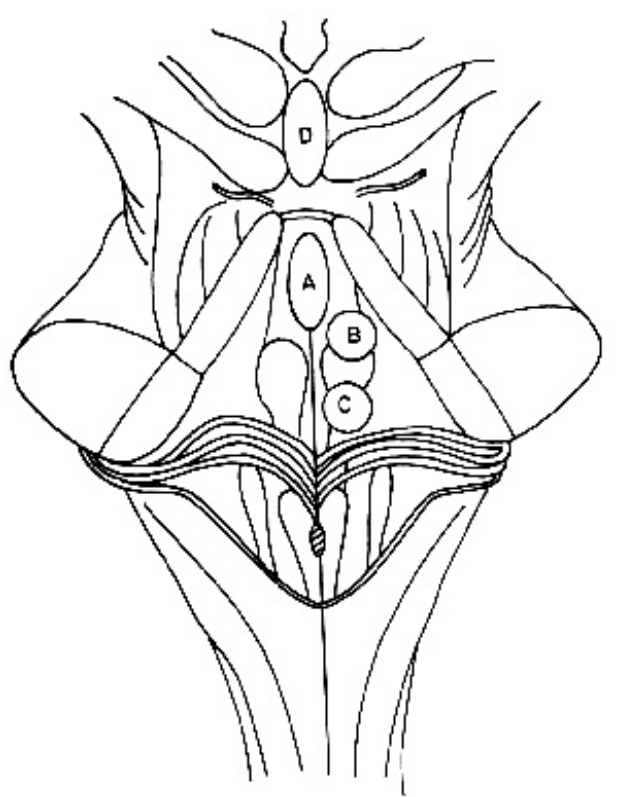

A. Midline above facial colliculus B. Suprafacial

C. Infrafacial

D. Intercollicular

Figure 4. Drawing showing the safe sites of incision on the dorsum of brain stem.

abscess is often difficult. Therefore, we believe that surgical aspiration with or without excision are necessary to identify the pathogen for successful treatment of patients.

Prior to the advent of the CT scan, early diagnosis and management of brain stem abscess constituted a formidable challenge. Patients with brain stem abscess most commonly present with cranial nerve deficits, especially facial weakness, dysphagia and abducens palsy, hemiparesis, headache and fever (15). However, classical brain stem syndromes that would allow a precise anatomical localization are rather infrequent. Moreover, the clinical picture is often complicated by co-existing basal meningitis and dural thrombophlebitis. The clinical course has been rapidly progressive deterioration ending uniformly in death, usually within a week or two after the onset of symptoms (15). This is primarily because their anatomic location can lead to catastrophic neurological complications and frequently preclude definitive drainage (16). Our two patients had clinical presentation of brain stem involvement in the form of $\mathrm{VI}^{\text {th }}$ and $\mathrm{VII}^{\text {th }}$ nerve paresis, hemiparesis, and with or without lower cranial nerve involvement. The third child manifested with severe neurological sequale reflecting the involvement of whole brain stem, probably more because of tuberculoma and less due to medullary abscess.

With cranial CT scan, identification and localization of intra-axial brain stem lesions have been greatly facilitated. On CT abscess in the brain stem has, similar characteristics as elsewhere in the brain, a fairly characteristic appearance consisting of central hypodensity surrounded by a smooth ring enhancement after intravenous contrast injection $(11,17)$. Our patients also presented with the similar CT findings, but with relatively thick ring in two cases in comparison to pyogenic abscesses as reported by us previously (11).

Various treatment protocols have been successfully tried till now like medical therapy alone (18), stereotactic aspiration along with medical therapy $(4,19)$, and microsurgical excision/drainage $(11,20)$. The major problem with microsurgical drainage is the incision related injury to major cranial nerves, while operating on dorsal brain stem through transventricular route. Moreover with the description of safe entry zones to the floor of $\mathrm{IV}^{\text {th }}$ ventricle, the morbidity in these cases is reduced. The incisions can be made at following levels to avoid major injuries; intercollicular incision, midline incision above facial colliculus, in suprafacial and infrafacial triangle (21). We tried to recognize these landmarks and availed them for successful drainage of abscess with relatively less morbidity (Figure 4). Though at times it is difficult to identify these landmarks with distorted anatomy.

The question of the optimal treatment for brain stem abscess remains open. The lack of an accurate bacteriological diagnosis is a reason of uncertainty regarding the outcome of conservative management alone. Two of our cases have characteristics similar to pyogenic abscesses. The treatment in these children would have gone futile without proper diagnosis. Progressive neurological deterioration and appearance of fresh lesions in temporal lobe and brain stem in our third child, despite regular intake of three drugs antituberculosis regimen, warranted surgical excision of tuberculoma and drainage of medullary abscess, as mentioned in literature also $(5,9)$. It is because tuberculous abscess are known to develop during course of antituberculosis chemotherapy warranting surgical excision (22). A trial of antimicrobial/antituberculosis treatment may be justified as long as the patient's clinical condition is stable on close neurological observation with CT monitoring if available. However, surgical evacuation of the pus should be carried out without delay at the first sign of clinical deterioration or CT evidence of enlargement of abscess.

\section{Conclusion}

Although CT stereotaxy and ultrasound guided needle aspiration seem to be the less invasive procedures, open operation and drainage of abscess under direct vision is not necessarily associated with a higher mortality/morbidity. It can be stated that proper microsurgical anatomical understanding 
of the brain stem and the selection of appropriate surgical approach is important for safe drainage of the abscess. Microsurgical drainage of abscess by safe incision in the floor of $\mathrm{IV}^{\text {th }}$ ventricle may avoid major morbidity.

Though tuberculosis has a definitive antimicrobial therapy. In spite of this brain stem tuberculous abscess need surgical drainage because medical therapy without accurate diagnosis is not efficient to treat the catastrophic presentation and the cases of paradoxical response to antituberculosis chemotherapy.

\section{Acknowledgement}

The authors are extremely grateful to Mr. A.P. Dhar Dwivedi for preparation of this manuscript.

\section{References}

1. Harvey FH, Carlow TJ. Brain stem abscess and the syndrome of acute tegmental encephalitis. Ann Neurol 1980; 7: 371-376.

2. Heilpern KL, Lorber B. Focal intracranial infections. Infect Dis Clin North Am 1996; 10: 879-898.

3. Schliamser SE, Backman K, Norrby SR. Intracranial abscess in adults : an analysis of 54 consecutive case. Scand J Infect Dis 1988; 20: 1-9.

4. Fuentes S, Bouillot P, Regis J, Lena G, Choux M. Management of brain stem abscess. Br J Neurosurg 2001; 15: 57-62.

5. Jamjoom ZA. Solitary brainstem abscess successfully treated by microsurgical aspiration. $\mathrm{Br}$ J Neurosurg 1992; 6: 249-253.

6. Rajshekhar V, Chandy MJ. Successful stereotactic management of a large cardiogenic brain stem abscess. Neurosurgery 1994; 34: 368-371.

7. Miller ES, Dias PS, Uttley D. CT scanning in the management of intracranial abscess: a review of 100 cases. Br J Neurosurg 1988; 2: 439-446.

8. Whitener DR. Tuberculous brain abscess. Report of a case and review of the literature. Arch Neurol 1978; 35: 148-155.

9. Kumar R. Atypical response to chemotherapy in neurotuberculosis. Br J Neurosurg 1998; 12: 344-
348.

10. Reichenthal E, Cohen ML, Schujman CB, Eynan N, Shalit M. Tuberculous brain abscess and its appearance on computerized tomography. J Neurosurg 1982; 56: 597-600.

11. Kumar R, Pandey CK, Bose N, Sahay S. Tuberculous brain abscess: clinical presentation, pathophysiology and treatment. (in children). Child's Nerv Syst 2002; 18: $118-123$.

12. Folgueira L, Delgado R, Palenque E, Noriega AR. Polymerase chain reaction for rapid diagnosis of tuberculous meningitis in AIDS patients. Neurology 1994; 44: 1336-1338.

13. Morgan H, Wood MW, Murphey F. Experience with 88 consecutive cases of brain abscess. J Neurosurg 1973; 38: 698-704.

14. Fulgham JR, Wijdicks EF, Wright AJ. Cure of a solitary brainstem abscess with antibiotic therapy: case report. Neurology 1996; 46: 1451-1454.

15. Weickhardt GD, Davis RL. Solitary abscess of brainstem. Neurology 1964; 14: 918-925.

16. Berg B, Franklin G, Cuneo R, Boldrey E, Strimling B. Neurosurgical cure of brain abscess: early diagnosis and follow-up with computerized tomography. Ann Neurol 1978; 3: 474-478.

17. Dake MD, McMurdo SK, Rosenblum ML, BrantZawadzki M. Pyogenic abscess of the medulla oblongata. Neurosurgery 1986; 18: 370-372.

18. Garcia-Monco JC, Gomez Beldarrain M, Fernandez Canton G, Capelastegui A, Collazos J. Resolution of a brainstem abscess through antituberculous therapy. Neurology 1997; 49: 265-267.

19. Nakajima $H$, Iwai $Y$, Yamanaka K, Kishi $H$. Successful treatment of brainstem abscess with stereotactic aspiration. Surg Neurol 1999; 52: 445 448.

20. Sarma S, Sekhar LN. Brain-stem abscess successfully treated by microsurgical drainage: a case report. Neurol Res 2001; 23: 855-861.

21. Choux M, Lena Gand DOL. Brain stem tumours. In: Choux M, Di Rocco C, Hockley AD, Walker M (eds). Textbook of Pediatric Neurosurgery $\left(1^{\text {st }} \mathrm{ed}\right)$. London: Churchill Livingstone, 1999, p 481

22. Prakash B, Mehta G, Gondal R, Kumar S, Malhotra V. Tuberculous abscess of the brain stem. Surg Neurol 1989; 32: 445-448. 